\title{
Electrospun fiber membranes enable proliferation of genetically modified cells
}

\author{
This article was published in the following Dove Press journal: \\ International Journal of Nanomedicine \\ 26 February 2013 \\ Number of times this article has been viewed
}

\author{
Mandula Borjigin* \\ Chris Eskridge* \\ Rohina Niamat \\ Bryan Strouse \\ Pawel Bialk \\ Eric B Kmiec \\ Department of Chemistry, Delaware \\ State University, Dover, DE, USA \\ *These authors contributed equally \\ to this work
}

\begin{abstract}
Polycaprolactone (PCL) and its blended composites (chitosan, gelatin, and lecithin) are well-established biomaterials that can enrich cell growth and enable tissue engineering. However, their application in the recovery and proliferation of genetically modified cells has not been studied. In the study reported here, we fabricated PCL-biomaterial blended fiber membranes, characterized them using physicochemical techniques, and used them as templates for the growth of genetically modified HCT116-19 colon cancer cells. Our data show that the blended polymers are highly miscible and form homogenous electrospun fiber membranes of uniform texture. The aligned PCL nanofibers support robust cell growth, yielding a 2.5 -fold higher proliferation rate than cells plated on standard plastic plate surfaces. PCL-lecithin fiber membranes yielded a 2.7-fold higher rate of proliferation, while PCL-chitosan supported a more modest growth rate (1.5-fold higher). Surprisingly, PCL-gelatin did not enhance cell proliferation when compared to the rate of cell growth on plastic surfaces.
\end{abstract}

Keywords: nanofibers, PCL-biomaterial blends, miscibility, gene editing, cell proliferation

\section{Introduction}

It is becoming increasingly apparent that biomaterials have an important role to play in the development and evolution of regenerative medicine. The fabrication of simple or complex matrices with secondary and tertiary structures is rapidly becoming key in nanotechnology approaches to human cell therapy. ${ }^{1,2}$ Perhaps not surprisingly, human cells appear to proliferate robustly on nanostructures, and this phenomenon can afford the opportunity for detailed evaluation of cellular growth mechanisms. ${ }^{3-5}$

The central objective of fabricating nanostructures for biological applications is the creation of compatible and biodegradable three-dimensional scaffolds upon which human cells can either differentiate, divide, or both. Polycaprolactone (PCL), a hydrophobic polymer, is a well-known biocompatible polymer on which to grow human cells and PCL nanofiber scaffolds can be produced by electrospinning. ${ }^{6-9}$ The nanofiber scaffolds are particularly useful because of their high surface area and porosity, which stimulates cell adhesion. Modifications and blending of PCL with other chemicals or proteins can create environments that can become even more conducive to cell growth. Among the more prominent sources of natural biomaterials are gelatin, chitosan, and lecithin, all of which can enhance the hydrophilicity and biocompatibility of PCL. ${ }^{10-15}$ Gelatin is composed of glycine, proline, and hydroxyproline, which act together to increase cell attachment. Nanofibers fabricated from a PCL-gelatin blend enhance cell adhesion and neurite outgrowth. ${ }^{16,17}$ Chitosan, derived from the deacetylation of chitin and composed of linear polysaccharides linked by $\beta(1-4)$-linked D-glucosamine,
Correspondence: Eric B Kmiec Department of Chemistry, Delaware State University, 1200 N DuPont Highway, Dover, DE 1990I, USA

Tel +l 3028576530

Fax +l 3028576539

Email ekmiec@desu.edu 
is well known for its capacity to support cell growth and expansion. ${ }^{13,14}$ Again, scaffolds prepared from a PCL-chitosan blend support excellent cell growth. ${ }^{18,19}$ Lecithin is composed of a complex mixture of phospholipids that contain two long carbon chains derived from soybean oils. ${ }^{20}$ Due to its amphiphilic chemical features, which are similar to the phospholipid components of the cell membrane, lecithin can serve as a superior biocompatible support material for cell attachment and growth. Lecithin has been successfully electrospun to fabricate fiber membranes ${ }^{21-23}$ and the fiber made from a PCL-lecithin blend has been used to construct ureteral ${ }^{15}$ and vascular ${ }^{24}$ grafts in murine animal models. In addition, a three-dimensional material with a porous structure fabricated from a lecithin-poly (L-lactic acid) blend was used in a rat bone-graft study, ${ }^{25}$ demonstrating improved hydrophilicity and biocompatibility. Hence, blending these biomaterials with PCL seems to provide an environment conducive to growth for cell differentiation and tissue engineering.

Investigations are underway with the goal of utilizing nanofiber templates that mimic biological structures to enable proliferation of stem cells in vitro. ${ }^{5,7,12}$ It is believed that, as nanofibers resemble an extracellular matrix, at least structurally, they can provide multilayered physical support for stem cell adhesion. ${ }^{26,27}$ While this approach has provided dynamic and exciting results for hematopoietic stem cells, ${ }^{5}$ neuronal stem cells, ${ }^{28}$ and mesenchymal stem calls, ${ }^{7,29}$ relatively little is known about how such nanofiber scaffolds enable the recovery and re-ignition of the proliferation of genetically modified human cells. These cells are particularly relevant to nanomedicine because they are the product of ex vivo gene therapy approaches.

The emergence of several techniques that can direct the correction of point mutations in human genes through the process of gene editing has provided real hope for ex vivo therapy of inherited diseases such as sickle cell disease. The active components of these techniques, oligonucleotides, zinc finger nucleases, and transcription activator-like effector nucleases, ${ }^{30-32}$ must be delivered into the target cell at levels that can have unintended effects - for example, zinc finger nuclease-offsite targeting. ${ }^{33,34}$ One documented side effect is the phenomenon known as "reduced proliferation phenotype,"35 in which cells that have undergone genetic modification have inherently reduced rates of replication and proliferation. ${ }^{36}$ This barrier needs to be removed before clinical applications of nanomedicine are designed and realized.

Recently, Borjigin et $\mathrm{al}^{37}$ showed that a single type of electrospun nanofiber can be used as a scaffold upon which genetically modified (gene-edited) cells can reignite the process of DNA replication and enable cell growth. These data suggest for the first time that electrospun PCL fibers have the capacity to enable the growth of human cells that bear a genetic change.

In the work reported here, we fabricated fiber membranes from PCL-biomaterial blends, examined their composition and miscibility, and evaluated their capacity to potentiate recovery and proliferation of genetically modified cells.

\section{Materials and methods Materials}

PCL (MW 80,000), chitosan (MW mid-size), and gelatin (type A) were purchased from Sigma-Aldrich (St Louis, MO, USA). Lecithin was purchased from TCI America (Portland, OR, USA). Dichloromethane (DCM), chloroform, and trifluoroethanol were purchased from Acros Organics (Monroeville, NJ, USA). N,N-Dimethylformamide and trifluoroacetic acid were purchased from Thermo Fisher Scientific (Waltham, MA, USA). and were used without further purification. HyClone culture medium was purchased from Fisher Scientific. The HCT116-19 cell line was constructed in one author's (EBK) laboratory by incorporating the enhanced green fluorescent protein (eGFP) mutant gene into the wild-type HCT116 (American Type Culture Collection, Manassas, VA, USA) genome.

\section{Electrospinning}

PCL solutions $(10 \%-20 \%)$ were made by dissolving PCL pellets in DCM and N,N-Dimethylformamide $(8: 2)$ according to the published procedures. ${ }^{38}$ The PCL fibers were fabricated using an electrospinning apparatus (described in Borjigin et al) ${ }^{37}$ setting the voltage at $12.5 \mathrm{kV}$, flow rate of the solution at $0.25 \mathrm{~mL} / \mathrm{h}$, and the distance between the spinneret and the collector at $10 \mathrm{~cm}$.

The PCL-chitosan solution was prepared by mixing $1.5 \mathrm{~mL} \mathrm{20 \%} \mathrm{PCL} \mathrm{solution} \mathrm{and} 0.5 \mathrm{~mL} \mathrm{7 \%}$ chitosan solution (made by dissolving chitosan powder in trifluoroacetic acid and DCM at a ratio 7:3, as described by Dhandayuthapani et al $)^{13}$ under sonication for 3 hours. Following this, PCLchitosan fibers were fabricated by electrospinning the blended polymers with the voltage set at $12.5 \mathrm{kV}$, a flow rate of $0.35 \mathrm{~mL} / \mathrm{h}$, and a distance between spinneret and gap collector of $10 \mathrm{~cm}$.

The PCL-gelatin solution was prepared by blending equal volumes of $15 \%$ PCL and $10 \%$ gelatin. The gelatin solution was made by dissolving the powder in trifluoroethanol as described by Gupta et al. ${ }^{17}$ The electrospinning parameters 
for the fabrication of PCL-gelatin fibers were a voltage of $12.5 \mathrm{kV}$, flow rate of $0.20 \mathrm{~mL} / \mathrm{h}$, and distance between the spinneret and the collector of $10 \mathrm{~cm}$.

Lastly, the PCL-lecithin solution was prepared by mixing an equal volume of $10 \%$ PCL and $80 \%$ lecithin and vortexing. The $80 \%$ lecithin was made by dissolving lecithin in chloroform and DCM (7:3) as described by McKee et al. ${ }^{21}$ The electrospinning parameters were a voltage of $15 \mathrm{kV}$, flow rate of $0.30 \mathrm{~mL} / \mathrm{h}$, and distance between spinneret and collector of $10 \mathrm{~cm}$.

\section{Characterization of electrospun fibers}

Scanning electron microscopy (SEM) was used to visualize the texture of the fiber membranes and to measure the diameter of the electrospun fibers. The fiber membranes were placed on an aluminum mount-M4 (cat \# 75610) (Electron Microscopy Sciences, Hatfield, PA, USA) and coated with $\mathrm{Au} / \mathrm{Pt}$ using a Denton Bench Top Turbo III carbon evaporator (Denton Vacuum, Moorestown, NJ, USA) prior to imaging under a Hitachi S4700 Field Emission Scanning Electron Microscope (Hitachi High Technologies, Tokyo, Japan) with magnification between 2500 and 70,000×. The fiber diameters were measured using ImageJ software (v 1.4; National Institute of Health, Bethesda, MD, USA). Eight fibers from each sample were measured, and the average and standard deviation were calculated.

A Nicolet 6700 FT-IR Spectrometer (Thermo Fisher Scientific, Waltham, MA, USA) was used to perform Fourier transform infrared spectroscopy (FTIR) using air as the (blank) control to determine the composition of the each fiber membrane by detecting the characteristic absorption band patterns associated with the rotational, rocking, twisting, and scissoring of the specific bonds within the fibers. The FTIR analysis was carried out over a wave number range between 4000 and $400 \mathrm{~cm}^{-1}$ at a resolution of $4 \mathrm{~cm}^{-1}$.

The miscibility of the blended polymers in the electrospun fiber membranes was investigated using X-ray diffraction (XRD) and differential scanning calorimetry (DSC). XRD was used to determine the crystalline structure and amorphousness of the electrospun fibers. The fiber samples were analyzed using an X-ray diffractometer (X-pert PW3040, PANalytical, Lelyweg, The Netherlands), operating at $40 \mathrm{kV}$ and $20 \mathrm{~mA}$ with a $\mathrm{Cu}-\mathrm{K} \alpha$ source. The diffraction intensity was measured in the range of $2 \theta$ angles between $10^{\circ}$ and $30^{\circ}$ with a scanning rate of $5^{\circ} / \mathrm{min}$. DSC analysis was performed on fiber samples ( $\sim 5 \mathrm{mg}$ ) using a Pyris Diamond TGA/DGA High Temperature 115 thermogravimetric/ differential analyzer (PerkinElmer, Waltham, MA, USA). The temperature was increased from ambient to $500^{\circ} \mathrm{C}$ in an argon atmosphere at a constant heating rate of $5^{\circ} \mathrm{C} / \mathrm{min}$.

\section{Cell recovery and proliferation on fiber membranes}

The integrated mutant eGFP gene in HCT116-19 cells was corrected by means of a standard gene-editing protocol. ${ }^{35-37}$ Briefly, cells were synchronized with $6 \mu \mathrm{M}$ aphidicolin followed by 4 hours of release prior to introduction of the $3^{\prime}$ PTO (phosphorothioate)-modified 72-nucleotide (NT) singlestranded oligodeoxynucleotide (ssODN) by electroporation. One million cells in $100 \mu \mathrm{L}$ of Hyclone McCoy's 5A serum free medium (Thermo Scientific, Logan, UT, USA) were mixed with the ssODN $(4 \mu \mathrm{M})$ in an electroporation cuvette with a 4 mm gap (Fisher Scientific, Hampton, NH, USA). The cells were electropermealized using a Gene Pulser Xcell ${ }^{\mathrm{TM}}$ electroporation apparatus (Bio-Rad, Hercules, CA, USA) with settings of $250 \mathrm{~V}, 13 \mathrm{~ms}, 2$ pulses, and a 1-second interval for delivery of ssODN into the cells. Immediately after the electroporation, the cells were transferred onto nanofibers for recovery and growth. Triplicate samples of cells recovered on fiber membranes were harvested at Day 4 (96 hours) and Day 7 (168 hours) for analysis of gene editing using fluorescence-activated cell-sorting (FACS) analysis (Guava EasyCyte HT, Millipore, Billerica, MA, USA). The corrected cells on the fibers at Day 4 (96 hours) were visualized and images were taken using an EVOS FL microscope (AMG Micro, Bothell, WA, USA). ${ }^{37}$

\section{Results \\ Electrospun PCL-biomaterial blended fibers}

Four different fibers were electrospun to fabricate parallelaligned fiber membranes using a standard electrospining apparatus setup, as described in "Materials and methods" (see Borjigin et al). ${ }^{37}$ A 21 -gauge flat-tip syringe needle was used as a spinneret and a gap collector was used to electrospin the fibers. The distance between the spinneret - from where the polymer solution spins out - and the collector was set $10 \mathrm{~cm}$ for the fabrication of all four fiber membranes. The flow rate of polymer solutions from the spinneret and the voltage gap applied between the spinneret and the collector were optimized for the specific polymer fiber fabrication. A flow rate of $0.25 \mathrm{~mL} / \mathrm{h}$ and voltage of $12.5 \mathrm{kV}$ were used in PCL nanofiber electrospinning; $0.35 \mathrm{~mL} / \mathrm{h}$ and $12.5 \mathrm{kV}$, $0.20 \mathrm{~mL} / \mathrm{h}$ and $15 \mathrm{kV}$, and $0.30 \mathrm{~mL} / \mathrm{h}$ and $15 \mathrm{kV}$ were applied in electrospinning PCL-chitosan, PCL-gelatin, and PCL-lecithin, respectively. The morphology and texture of 
fiber membranes fabricated from pure PCL polymer were more uniform and the fibers were aligned more neatly than in the blended polymers. The diameter of a PCL nanofiber was determined to be $428 \pm 40 \mathrm{~nm}$ (Figure 1A), while that of a PCL-chitosan nanofiber was thinner $(113 \pm 31 \mathrm{~nm})$ and exhibited a rougher surface (Figure 1B). The two other electrospun blend fibers, PCL-gelatin and PCL-lecithin, had a diameter of $3.20 \pm 1.65 \mu \mathrm{m}$ and $1.80 \pm 0.90 \mu \mathrm{m}$, respectively (Figure $1 \mathrm{C}$ and D).

\section{Composition of blended fibers}

FTIR analysis was conducted using a Nicolet 6700 FT-IR spectrometer to examine the composition of fiber membranes and to determine the presence of each component in the miscible blended fibers. FTIR was performed using air as a blank control over the range of 4000-400 wavenumber/ $\mathrm{cm}$ at a resolution of $4 \mathrm{~cm}^{-1}$. PCL nanofibers exhibited a characteristic band at $1160 / \mathrm{cm}$ in the fingerprint range of the FTIR absorption spectrum; this is attributed to $\mathrm{C}-\mathrm{O}-\mathrm{C}$ bond stretching within the PCL polymer. In addition, a very strong carbonyl group bond stretching at $1720 / \mathrm{cm}$ and C-H stretching at 2860 and $2940 / \mathrm{cm}$ were detected in the PCL nanofibers (Figure 2). This spectrum pattern was also present in all three blended fibers, albeit at weaker intensity, signifying the presence of PCL in the blended polymers. The PCL-chitosan blend (10\% PCL, 3.5\% chitosan in polymer solution, comprising 10:3.5 ratio in the dry fibers) nanofibers exhibited a unique band at $1540 / \mathrm{cm}$, indicating $\mathrm{N}-\mathrm{H}$ stretching from the primary amine of chitosan. PCL-gelatin
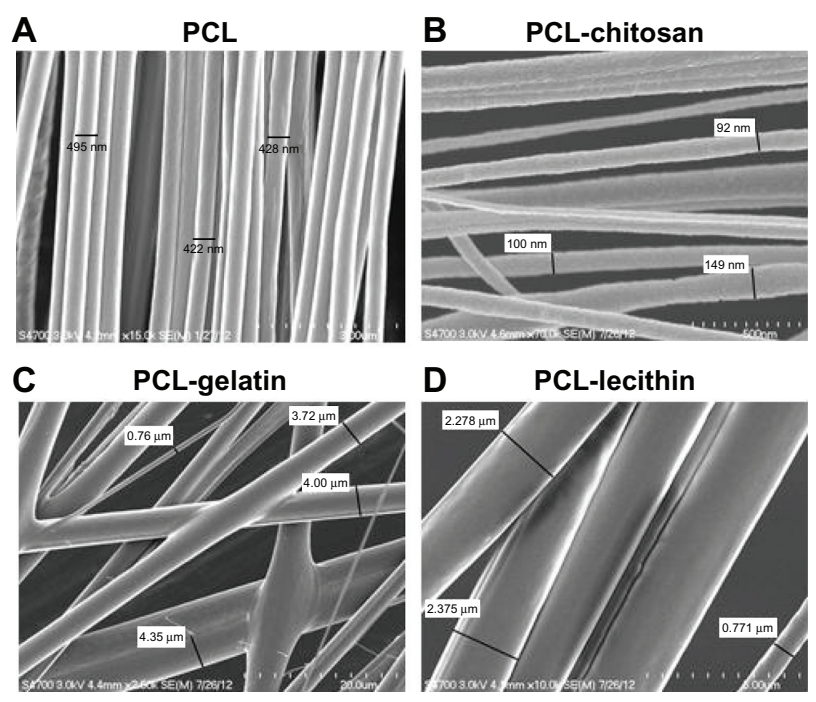

Figure I Scanning electron microscope (SEM) images of parallel-aligned electrospun fibers: (A) polycaprolactone (PCL), (B) PCL-chitosan, (C) PCL-gelatin, and (D) PCL-lecithin.

Note: Eight fibers observed in each sample were measured and the average and standard deviation were calculated.

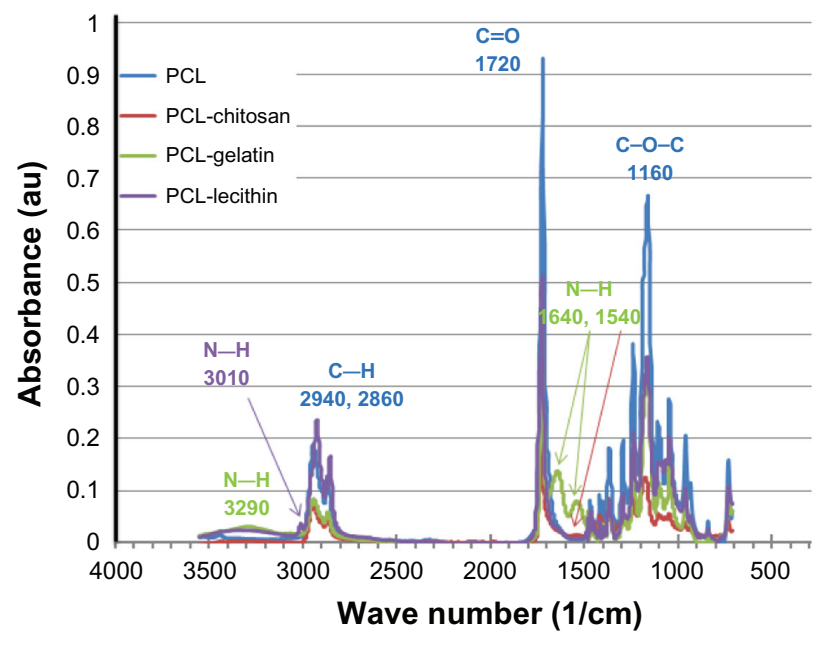

Figure 2 Fourier transform infrared spectroscopy spectra of the fiber membranes. Abbreviation: $\mathrm{PCL}$, polycaprolactone.

(7.5\% PCL and 5\% gelatin, ratio of dry weight 7.5:5) fibers showed characteristic bands at 1540, 1640, and 3290/cm, signaling $\mathrm{N}-\mathrm{H}$ stretching from the primary and secondary amines from peptides and proteins inherent in the gelatin mix. Finally, PCL-lecithin (5\% PCL, 40\% lecithin, weight ratio of 5:40) fibers exhibited a weak band at $3010 / \mathrm{cm}$, indicating $\mathrm{N}-\mathrm{H}$ stretching in the quaternary amine group of lecithin phosphatidylcholine (Figure 2). Our FTIR absorption spectra of PCL, PCL/chitosan, and PCL/gelatin are consistent with previously published data. ${ }^{13,16,17}$ Our data confirm the presence of the indicated natural materials in the blended membranes at the predicted composition.

\section{XRD and DSC}

XRD of the fibers was carried out using the X-pert PW3040 diffractometer (PANalytical) operating at $40 \mathrm{kV}$ and $20 \mathrm{~mA}$ with a $\mathrm{Cu}-\mathrm{K} \alpha$ source. The PCL nanofibers exhibited two strong diffraction peaks at Bragg angles $2 \theta=21.3^{\circ}$ and 23.6 which represent the (110) and (200) reflections respectively of a polyethylene-like crystal structure with orthorhombic unit cell parameters ${ }^{8,39,40}$ (see Figure 3). The PCL-chitosan nanofiber displayed a much weaker diffraction at $2 \theta=20.2^{\circ}$ and $22.4^{\circ}$, shifting one degree left of the PCL nanofiber peaks, indicating a different type of crystal form with different D spacing within the crystal lattice. PCL-gelatin fiber diffracted at $2 \theta=22.8^{\circ}$ and $24.7^{\circ}$ (right shift) with a much weaker intensity than that of the pure PCL nanofibers, indicating a different crystal form and a less ordered structure or a lesser degree of crystallinity in the blend material. Lastly, PCL-lecithin showed moderate intensity diffractions at $2 \theta=20.8^{\circ}, 21.7^{\circ}, 23.1^{\circ}$, and $24.1^{\circ}$, indicating that different forms of crystals were present in this blended material 


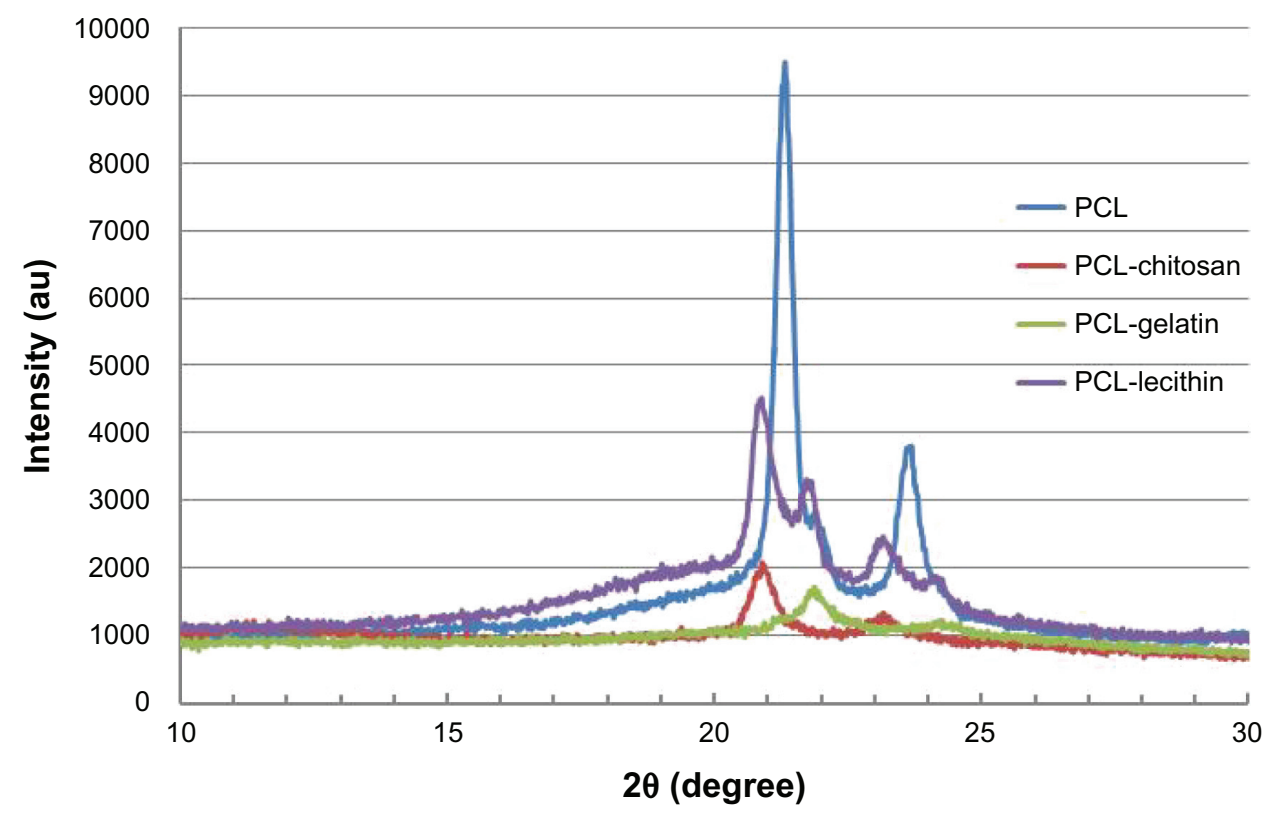

Figure 3 X-ray diffraction patterns of the fiber membranes.

Notes: Polycaprolactone (PCL) nanofibers exhibit two strong diffraction peaks at Bragg angles $2 \theta=21.3^{\circ}$ and $23.6^{\circ}$. PCL-chitosan shows much weaker diffraction at $2 \theta=22.2^{\circ}$ and $22.4^{\circ}$, while PCL-gelatin is weaker again at $2 \theta=22.8^{\circ}$ and $24.7^{\circ}$. PCL-lecithin shows moderate intensity diffraction at $2 \theta=20.8^{\circ}, 21.7^{\circ}, 23.1^{\circ}$, and $21.7^{\circ}$.

(Figure 3). These data demonstrate that crystal forms and the orientation in the blended polymer fibers are different from those found in pure PCL nanofiber membranes. These results further indicate that the transparent and visually miscible polymer solutions of blended PCL-biomaterials formed amorphous composite fibers with different types of crystalline structure.

To further examine whether the homogeneity and miscibility of the blended fibers were affected by the lesser degree of crystallinity or the different forms of crystals in the fibers, DSC was conducted using the Pyris Diamond thermogravimetric/differential analyzer. Theoretically, a completely miscible blend should result in the formation of a single phase-transition temperature rather than the separate phase transition of individual parent polymers. Fiber samples $(5 \mathrm{mg})$ were sealed in aluminum pans of the instrument and the pan was heated from ambient temperature to $500^{\circ} \mathrm{C}$ at $5^{\circ} \mathrm{C} / \mathrm{min}$ increments under argon purge at a $20 \mathrm{~mL} / \mathrm{min}$ rate. The analysis showed that the PCL nanofiber has an onset temperature of $52.6^{\circ} \mathrm{C}$, at which point the PCL nanofiber starts transitioning to a liquid state, with a melting point of approximately $61^{\circ} \mathrm{C}$. Noticeably, the three natural polymers (chitosan, gelatin, and lecithin) blended into PCL led to an increase in the melting temperature. The onset temperature of the PCLchitosan composite nanofibers was $57.2^{\circ} \mathrm{C}$, and its melting point was $65.3^{\circ} \mathrm{C}$; the PCL-gelatin composite fibers showed an onset temperature of $58.4^{\circ} \mathrm{C}$ and melting point at $64.7^{\circ} \mathrm{C}$; and the PCL-lecithin fibers displayed an onset temperature of $55.5^{\circ} \mathrm{C}$ and a melting point of $63.2^{\circ} \mathrm{C}$.

In contrast, the depolymerization temperature of pure PCL nanofibers was higher than that of the three blended PCL-biomaterial fibers. The depolymerization of PCL nanofibers occurred at a temperature of $389^{\circ} \mathrm{C}$, whereas the depolymerization of PCL-chitosan nanofibers occurred at $368^{\circ} \mathrm{C}$, that of PCL-gelatin fibers at $379^{\circ} \mathrm{C}$, and that of PCLlecithin fibers at $382^{\circ} \mathrm{C}$ (Figure 4). The single peak each for phase-transition temperature and decomposition temperature of pure PCL and blended natural polymers confirms that PCL blended into the three natural polymers forms highly miscible polymer fibers, albeit with different levels and types of crystallinity present.

\section{Recovery and proliferation of genetically modified cancer cells}

Gene editing in human cells consists of the molecular exchange of a nucleotide in the coding region of a gene, as directed by a ssODN. ${ }^{30,41-46}$ Largely, mechanistic studies have utilized a mutated $e G F P$ gene as the target and a functional eGFP is produced if the gene is repaired; then, the corrected cells express $e G F P$ and display green fluorescence. In the standard model system, a single copy of the $e G F P$ gene with a single base mutation $-\mathrm{TAC} \rightarrow \mathrm{TAG}$ - generating a stop codon has been stably integrated in the sequence of HCT116 cells. To correct the mutation, the appropriate oligonucleotide is introduced into the cell population by 


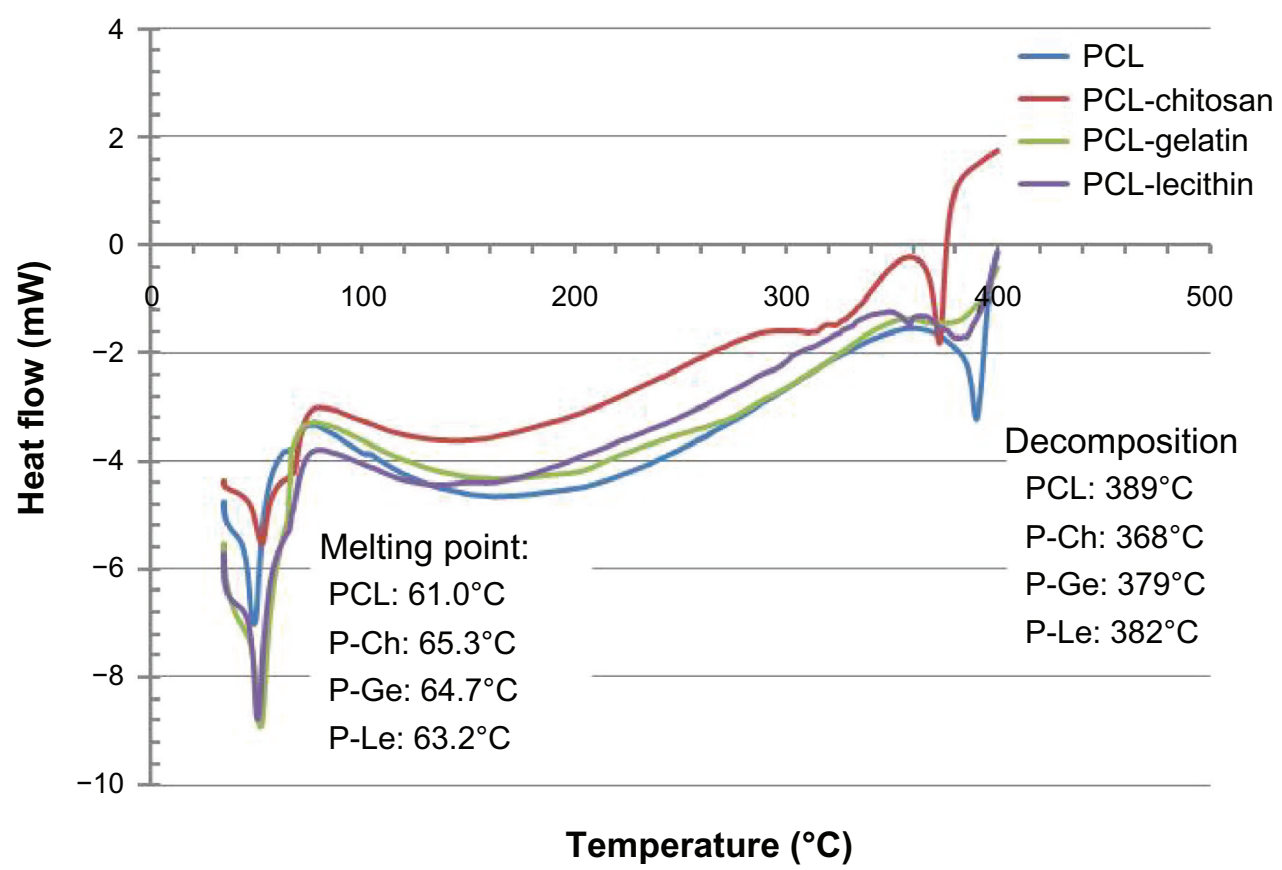

Figure 4 Differential scanning calorimetry thermograms of the fiber membranes.

Abbreviations: PCL, polycaprolactone; P-Ch, polycaprolactone-chitosan; P-Ge, polycaprolactone-gelatin; P-Le, polycaprolactone-lecithin.

electroporation. We have previously employed this type of system to evaluate gene editing in HCT116 cells. ${ }^{30,35,36}$ The method involves synchronizing HCT116-19 cells using aphidicolin for 24 hours followed by a 4-hour release period prior to introduction of 72 NT DNA oligonucleotides by electroporation (Figure 5A). Immediately after electroporation, $0.5 \times 10^{6}$ cells are plated onto a polylysinecoated dish or an electrospun fiber membrane for recovery and expansion. Due to the large number of free ends delivered into the cell, which is part of the ODN-directed gene-editing process, the DNA damage response checkpoint proteins CK1 and CK2 are activated and DNA replication is slowed or halted. As a result, the targeted cell appears to enter a quiescent state of growth. ${ }^{47,48}$ Such stress responses, along with the physical harm incurred by the process of electroporation itself, result in a long recovery phase, which we have termed the "reduced proliferation phenotype" (RPP). ${ }^{30,35,47,48}$

Since the three PCL-biomaterial-blended electrospun fiber membranes demonstrated excellent miscibility, we evaluated whether they were capable of supporting cell recovery and, essentially, can enable reversal of RPP. Our data show that corrected cells (green) grown on three of the electrospun fiber membranes, PCL, PCL-chitosan, and PCLlecithin, divide at Day 4 (96 hours) in contrast to cells plated onto the standard plastic surface or onto PCL-gelatin fiber membranes (Figure 5B). Triplicate samples of cells from each fiber membrane and the plastic surface were harvested at Day 4 (96 hours), and the correction efficiency was quantified using FACS. The correction efficiency of cells on PCL, PCLchitosan, and PCL-lecithin was comparable; approximately 1.8 and 1.9 times higher than that found for PCL-gelatin fiber membranes and plastic surfaces, respectively (see Figure 5C). These results suggest that a higher magnitude of cell recovery is enabled when cells are plated onto PCL, PCL-chitosan, and PCL-lecithin fiber membranes, and that RPP can be reversed.

Cell proliferation on the fiber membranes and plastic surfaces was also quantified at Day 7 (168 hours). PCLlecithin fiber membranes were found to promote cell growth the best, with a rate 3.5 times higher than that seen with PCLgelatin fiber membranes and 2.7 times higher than that seen with plastic surfaces. PCL and PCL-chitosan membranes enhanced the proliferation of the cells 3.2 and 1.9 times more than PCL-gelatin (Figure 5C). The correction efficiency of cells grown on PCL-gelatin fiber membranes declined precipitously at both time points, demonstrating that cell expansion on this fiber membrane was impeded.

\section{Discussion}

Electrospun nanofibers have attracted a great deal of attention for applications in tissue engineering due primarily to their topological similarity to the extracellular matrix, large surface to volume ratio, flexibility in surface functionality, 
A

Wild-type eGFP 5'GTGCCCTGGCCCACCCTCGTGACCACCCTGACCTACGGCGTGCAGTGCTTCAGCCGCTACCCCGACCACATG3' NT Mutant eGFP 5'GTGCCCTGGCCCACCCTCGTGACCACCCTGACCTAGGGCGTGCAGTGCTTCAGCCGCTACCCCGACCACATG3' NT

72 NT $\quad 3^{\prime}-C^{*} A^{*} C^{*} G G$ GACCGGGTGGGAGCACTGGTGGGACTGGATGCCGCACGTCACGAAGTCGGCGATGGGGCTGGTG* $T^{*} A^{*} C-5$

\begin{tabular}{|c|c|c|c|c|}
\hline Synchronize & Release & $\begin{array}{l}72 \text { NT delivery } \\
\text { and editing }\end{array}$ & $\begin{array}{l}\text { Recovery and } \\
\text { proliferation }\end{array}$ & FACS analysis \\
\hline $\begin{array}{l}\text { - Cells synchronized } \\
\text { at the } \mathrm{G} 1 / \mathrm{S} \text { border with } \\
6 \mu \mathrm{M} \text { aphidicolin for } \\
16-24 \text { hours }\end{array}$ & $\begin{array}{l}\text { Release from } \\
\text { cell-cycle arrest } \\
\text { for } 4 \text { hours, } \\
\text { allowing S-phase } \\
\text { to resume }\end{array}$ & $\begin{array}{l}\text { - } 4 \text { uM } 72 \text { NT delivered } \\
\text { into cells via } \\
\text { electroporation } \\
\text { - Gene-editing reaction } \\
\text { occurs }\end{array}$ & $\begin{array}{l}\text { - Cells immediately } \\
\text { plated onto dish } \\
\text { surface or fiber } \\
\text { membranes }\end{array}$ & $\begin{array}{l}\text { - Cells harvested } \\
\text { from dish surfaces } \\
\text { or fiber membranes } \\
\text { at } 96 \text { or } 168 \text { hours } \\
\text { after plating } \\
\text { - FACS analysis } \\
\text { performed }\end{array}$ \\
\hline
\end{tabular}

B

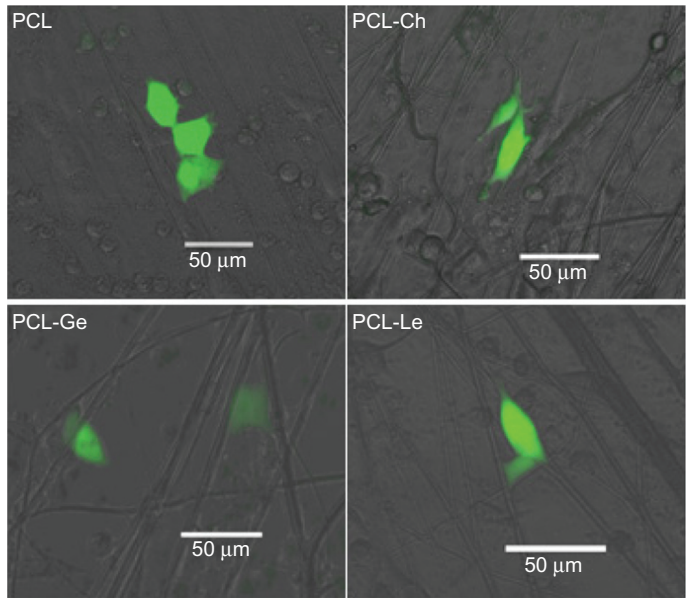

C

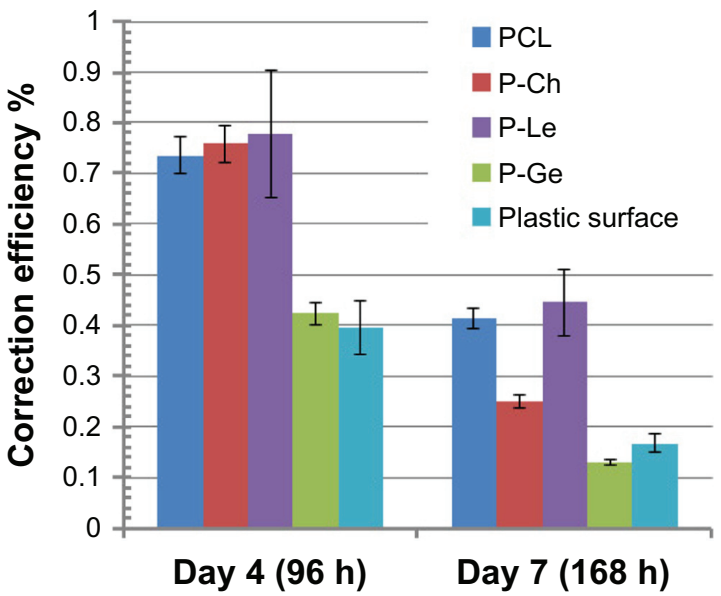

Figure 5 Recovery of the genetically modified cells on electrospun fiber membranes. (A) Model system and experimental protocol for gene editing. (B) Fluorescent microscope images of the genetically modified HCTII6-19 cells on fiber membranes at Day 4 (96 hours) using an EVOS FL microscope (AMG Micro, Bothell, WA, USA) at 170x magnification. (C) Gene correction efficiency of the cells on fiber membranes at Day 4 (96 hours) and Day 7 (168 hours) was analyzed using a Guava fluorescence-activated cell-sorting (FACS) machine (Millipore, Billerica, MA, USA).

Abbreviations: eGFP, enhanced green fluorescent protein; NT, nucleotide; PCL, polycaprolactone; P-Ch, polycaprolactone-chitosan; P-Ge, polycaprolactone-gelatin; P-Le, polycaprolactone-lecithin.

and adjustable porosity. ${ }^{4,49}, 50$ Recently, homogenous PCL nanofibers have been used to enable proliferation of genetically modified cells. ${ }^{37}$ Here, we extended that initial study and fabricated parallel-aligned PCL-chitosan, PCLgelatin, and PCL-lecithin electrospun fiber membranes with visually well-blended PCL-biomaterial solutions, and confirmed the presence of the expected components in the fiber membranes by FTIR. We examined the fiber quality (amorphous, ordered structure, or crystallinity) of the fibers by XRD and determined the miscibility of the components by DSC analysis. Then, these membranes were used as recovery scaffolds to expand genetically modified cells that are known not to proliferate on standard plastic surfaces.

Certain features of electrospun fibers, such as morphology, diameter, and density, are important to the creation of a conducive nanofiber environment for cell growth. ${ }^{50}$ The SEM data show that electrospun PCL-chitosan and PCL fibers were nanoscale, and PCL-gelatin fibers exhibit a larger diameter with higher deviation than other fibers (PCL, PCL-chitosan, and PCL-lecithin) (Figure 1). The fiber diameter variation was caused by ambient conditions (such as temperature and humidity), fluid properties, and electrospinning operating parameters. Fluid properties are viscosity, surface tension, boiling point, conductivity, and dielectric constant, and so forth. ${ }^{51,52}$ In our study, we used published protocols to fabricate the fiber membranes, but did not explore modulating these variables. The operating parameters are flow rate, voltage, and distance between the tip and the collector; these parameters were adjusted to produce the well-distributed defect-free fibers. 
The correction efficiency of HCT116-19 cells at Day 4 (96 hours) on PCL-gelatin was 1.8-fold lower than that on PCL, PCL-chitosan, or PCL-lecithin fiber membranes. Furthermore, the correction efficiency at Day 7 (168 hours) on PCL-gelatin fibers dropped 3.2- and 3.5-fold lower compared to PCL and PCL-lecithin, respectively (Figure 5C). After recovery on PCL-lecithin, PCL, and PCL-chitosan fiber membranes for 7 days (168 hours), the correction efficiency of HCT116-19 cells was 2.7-, 2.5-, and 1.5-fold higher, respectively, than that on standard plastic surfaces. PCL-lecithin membranes demonstrated the highest level of cell growth (along with pure PCL nanofiber membranes), while PCL-gelatin neither supported the recovery of cells up to Day 4 (96 hours) nor promoted the expansion of the cell growth until Day 7 (168 hours). Although the three blend biomaterials (chitosan, gelatin, and lecithin) provide conducive cell growth environments, as demonstrated in the literature, ${ }^{10-15}$ the level of recovery and expansion of our genetically modified cells varied. One reason for this variation is the cell-specific interaction with the biomaterials. Such interaction is perhaps more important to our genetically modified cells, since the process of genetic modification in our model system can affect cell metabolism, including the delivery of a large amount of ssODN into cells via electroporation.

Studies on other types of cells grown on various fibers have revealed that the fiber diameter plays an important role in cell growth. Cells cultured on fibers of larger diameter (micron size) tend to adhere to a single fiber and are seen not to proliferate. ${ }^{53}$ This observation is supported by the stronger growth of neural stem cells on nanofibers than on micro fibers. ${ }^{54}$ In contrast, a recent study by Cardwell et al showed that human mesenchymal stem cells proliferate and differentiate better on microfibers than on nanofibers. ${ }^{55}$ In our study, the correction efficiency on PCL-chitosan was comparably higher than those on PCL and PCL-lecithin during the recovery period (at Day 4), and it dropped significantly during the extended period. An explanation for this observation might be that a component released from the dissolved chitosan fiber interacts with cells to trigger a different pathway of physiology over the time. Therefore, the size of the fiber diameter might have a significant impact on cell attachment during the cell recovery period (4 days), while post-recovery cell physiology may be more dependent on the interaction between the cells and the biomaterials.

Not only is the miscibility of the blended polymers essential for formation of defect-free electrospun nanofibers, but it also provides uniform chemical properties that can enhance cell adhesion and proliferation. ${ }^{18,39}$ In our study, FTIR analyses show that the PCL nanofibers exhibit strong carbonyl group bond stretching at $1720 / \mathrm{cm}$ and $\mathrm{C}-\mathrm{H}$ stretching at 2860 and $2940 / \mathrm{cm}$, which matches exactly with the characteristic absorption bands presented in the literature. ${ }^{16,17}$ In addition, the blended fibers displayed unique peaks that can be attributed to the corresponding individual components. PCL-gelatin had several characteristic bands at 1540,1640 , and 3290/cm, attributed to N-H stretching from the primary and secondary amines in the peptide and protein components of the gelatin. PCL-chitosan nanofibers and PCL-lecithin fibers were distinguished from other blended fibers due to N-H stretching from the primary amine of chitosan at $1540 / \mathrm{cm}$ and a weak band at $3010 / \mathrm{cm}$ indicating $\mathrm{N}-\mathrm{H}$ stretching in the quaternary amine group of lecithin phosphatidylcholine (Figure 2).

The physical nature of the components (ie, miscibility, amorphous or ordered structure, and crystallinity) in the blended fibers was examined using XRD and DSC analysis. The XRD data revealed that pure PCL fibers formed a more ordered structure or exhibited higher crystallinity, while the blended fibers were more amorphous (with less crystallinity) and the PCL nanofibers were found to have a higher diffraction intensity (Figure 3). Our data also indicate that the blended biomaterials contributed to the formation of different types of crystals and crystal-plane orientations, interpreted from the $2 \theta$ angle shift (Figure 3). This indicates that the blended polymers were highly miscible and formed uniform composite materials. DSC analysis demonstrated that all three blended fibers and PCL differ in terms of both melting point and decomposition temperature, which again confirms the component difference in the blends. There is only a single phasetransition temperature (melting point) for each blended fiber membrane, further verifying that each blended fiber membrane was highly miscible (Figure 4).

Although the electrospun nanofiber scaffolds made from PCL, PCL-chitosan, and PCL-gelatin have been examined in cell differentiation studies, ${ }^{16,56,57}$ their application in the recovery and proliferation of genetically modified cells is, as far as the authors are aware, reported for the first time here. While PCL-lecithin is relatively less well-studied in cell growth and tissue engineering, it exhibits high potential for tissue grafts. ${ }^{15,24}$ In this study, PCL-lecithin supported the recovery and proliferation of genetically modified cells in a robust fashion (Figure 5B). In contrast, PCL-gelatin displayed no enhancement over plastic surfaces for recovery and proliferation. 


\section{Conclusion}

In our study, we fabricated electrospun fiber membranes using PCL, PCL-chitosan, PCL-gelatin, and PCL-lecithin, characterized them using chemical and physical methods, and applied them to the recovery and growth of genetically modified cells. Our data show that all the blended polymers formed miscible defect-free parallel-aligned fibers, and that PCL-lecithin and PCL were best at enhancing cell recovery and proliferation. In contrast, PCL-gelatin does not support the growth of genetically modified cells, and further study on this topic might reveal interesting mechanisms.

\section{Acknowledgments}

This work was supported by National Institutes of Health grants 5R01CA089325-12 and R21N5053608-03. We thank Dr Andrew Goudy, Dr Cherese Winstead, Dr Noureddine Melikechi, Mr Samuel Orefuwa, and Mr Adeola Ibikunle (Delaware State University) and Ms Debbie Powell from Bioimaging Center of Delaware Biotechnology Institute.

\section{Disclosure}

The authors report no conflicts of interest in this work.

\section{References}

1. Halberstadt C, Emerich DF, Gonsalves K. Combining cell therapy and nanotechnology. Expert Opin Biol Ther. 2006;6(10):971-981.

2. Romo-Herrera JM, Terrones M, Terrones H, Dag S, Meunier V. Covalent 2D and 3D networks from 1D nanostructures: designing new materials. Nano Lett. 2007;7(3):570-576.

3. Solanki A, Kim JD, Lee KB. Nanotechnology for regenerative medicine: nanomaterials for stem cell imaging. Nanomedicine. 2008;3(4): $567-578$.

4. Vasita R, Katti DS. Nanofibers and their applications in tissue engineering. Int J Nanomedicine. 2006;1(1):15-30.

5. Lu J, Aggarwal R, Pompili VJ, Das H. A novel technology for hematopoietic stem cell expansion using combination of nanofiber and growth factors. Recent Pat Nanotechnol. 2010;4(2):125-135.

6. Reneker DH, Kataphinan W, Theron A, Zussman E, Yarin AL. Nanofiber garlands of polycaprolactone by electrospinning. Polymer. 2002;43(25):6785-6794.

7. Li WJ, Tuli R, Okafor C, et al. A three-dimensional nanofibrous scaffold for cartilage tissue engineering using human mesenchymal stem cells. Biomaterials. 2005;26(6):599-609.

8. Yeo MG, Kim GH. Preparation and characterization of 3D composite scaffolds based on rapid-prototyped PCL/B-TCP struts and electrospun PCL coated with collagen and HA for bone regeneration. Chem Mater. 2012;24(5):903-913.

9. Venugopal J, Zhang YZ, Ramakrishna S. Fabrication of modified and functionalized polycaprolactone nanofibre scaffolds for vascular tissue engineering. Nanotechnology. 2005;16(10):2138-2142.

10. Zhang S, Huang Y, Yang X, et al. Gelatin nanofibrous membrane fabricated by electrospinning of aqueous gelatin solution for guided tissue regeneration. J Biomed Mater Res A. 2009;90(3): 671-679

11. Chong EJ, Phan TT, Lim IJ, et al. Evaluation of electrospun PCL/ gelatin nanofibrous scaffold for wound healing and layered dermal reconstitution. Acta Biomater. 2007;3(3):321-330.
12. Heydarkhan-Hagvall S, Schenke-Layland K, Dhanasopon AP, et al. Three-dimensional electrospun ECM-based hybrid scaffolds for cardiovascular tissue engineering. Biomaterials. 2008;29(19):2907-2914.

13. Dhandayuthapani B, Krishnan UM, Sethuraman S. Fabrication and characterization of chitosan-gelatin blend nanofibers for skin tissue engineering. J Biomed Mater Res B Appl Biomater. 2010;94(1):264-272.

14. Bhattarai N, Edmondson D, Veiseh O, Matsen FA, Zhang M. Electrospun chitosan-based nanofibers and their cellular compatibility. Biomaterials. 2005;26(31):6176-6184.

15. Shen J, Fu X, Ou L, et al. Construction of ureteral grafts by seeding urothelial cells and bone marrow mesenchymal stem cells into polycaprolactone-lecithin electrospun fibers. Int J Artif Organs. 2010;33(3):161-170.

16. Alvarez-Perez MA, Guarino V, Cirillo V, Ambrosio L. Influence of gelatin cues in PCL electrospun membranes on nerve outgrowth. Biomacromolecules. 2010;11(9):2238-2246.

17. Gupta D, Venugopal J, Prabhakaran MP, et al. Aligned and random nanofibrous substrate for the in vitro culture of Schwann cells for neural tissue engineering. Acta Biomater. 2009;5(7):2560-2569.

18. Sarasam A, Madihally SV. Characterization of chitosanpolycaprolactone blends for tissue engineering applications. Biomaterials. 2005;26(27):5500-5508.

19. Cooper A, Bhattarai N, Zhang M. Fabrication and cellular compatibility of aligned chitosan-PCL fibers for nerve tissue regeneration. Carbohydr Polym. 2011;85(1):149-156.

20. Naglič M, Smidovnik A, Koloini T. Kinetics of catalytic transfer hydrogenation of soybean lecithin. Ind Eng Chem Res. 1997;36(12): 5240-5245.

21. McKee MG, Layman JM, Cashion MP, Long TE. Phospholipid nonwoven electrospun membranes. Science. 2006;311(5759):353-355.

22. Park SA, Park KE, Kim WD. Preparation of sodium alginate/ poly(ethylene oxide) blend nanofibers with lecithin. Macromolecular Research. 2010;18(9):891-896.

23. Zhu N, Cui FZ, Hu K, Zhu L. Biomedical modification of poly(Llactide) by blending with lecithin. J Biomed Mater Res A. 2007;82(2): 455-461.

24. Zhang M, Wang K, Wang Z, Xing B, Zhao Q, Kong D. Small-diameter tissue engineered vascular graft made of electrospun PCL/lecithin blend. J Mater Sci Mater Med. 2012;23(11):2639-2648.

25. Wang Y, Cui FZ, Jiao YP, Hu K, Fan DD. Modification of bone graft by blending with lecithin to improve hydrophilicity and biocompatibility. Biomed Mater. 2008;3(1):015012.

26. Lim SH, Mao HQ. Electrospun scaffolds for stem cell engineering. Adv Drug Deliv Rev. 2009;61(12):1084-1096.

27. Keung AJ, Healy KE, Kumar S, Schaffer DV. Biophysics and dynamics of natural and engineered stem cell microenvironments. Wiley Interdiscip Rev Sys Biol Med. 2010;2(1):49-64.

28. Wang Y, Yao M, Zhou J, et al. The promotion of neural progenitor cells proliferation by aligned and randomly oriented collagen nanofibers through $\beta 1$ integrin/MAPK signaling pathway. Biomaterials. 2011;32(28):6737-6744.

29. Orza A, Soritau O, Olenic L, et al. Electrically conductive gold-coated collagen nanofibers for placental-derived mesenchymal stem cells enhanced differentiation and proliferation. ACS Nano. 2011;5(6): 4490-4503.

30. Engstrom JU, Suzuki T, Kmiec EB. Regulation of targeted gene repair by intrinsic cellular processes. Bioessays. 2009;31(2):159-168.

31. Urnov FD, Rebar EJ, Holmes MC, Zhang HS, Gregory PD. Genome editing with engineered zinc finger nucleases. Nat Rev Gen. 2010;11(9): 636-646.

32. Cermak T, Doyle EL, Christian M, et al. Efficient design and assembly of custom TALEN and other TAL effector-based constructs for DNA targeting. Nucleic Acids Res. 2011;39(12):e82.

33. Radecke S, Radecke F, Cathomen T, Schwarz K. Zinc-finger nuclease-induced gene repair with oligodeoxynucleotides: wanted and unwanted target locus modifications. Mol Ther. 2010;18(4):743-753. 
34. Lee HJ, Kweon J, Kim E, Kim S, Kim JS. Targeted chromosomal duplications and inversions in the human genome using zinc finger nucleases. Genome Res. 2012;22(3):539-548.

35. Bonner M, Kmiec EB. DNA breakage associated with targeted gene alteration directed by DNA oligonucleotides. Mutat Res. 2009;669(1-2): 85-94.

36. Bonner M, Strouse B, Applegate M, Livingston P, Kmiec EB. DNA damage response pathway and replication fork stress during oligonucleotide directed gene editing. Mol Ther Nucleic Acids. 2012;1:e18.

37. Borjigin M, Strouse B, Niamat RA, et al. Proliferation of genetically modified human cells on electrospun nanofiber scaffolds. Mol Ther Nucleic Acids. 2012;1:e59.

38. Xie J, MacEwan MR, Li X, Sakiyama-Elbert SE, Xia Y. Neurite outgrowth on nanofiber scaffolds with different orders, structures, and surface properties. ACS Nano. 2009;3(5):1151-1159.

39. Meng ZX, Zheng W, Li L, Zheng YF. Fabrication and characterization of three-dimensional nanofiber membrance of PCL-MWCNTs by electrospinning. Mater Sci Eng C. 2010;30:1014-1021.

40. Kim GH, Yoon H. Effect of an auxiliary electrode on the crystalline morphology of electrospun nanofibers. Appl Phys Lett. 2008;93(2):023127.

41. Papaioannou I, Disterer P, Owen JS. Use of internally nuclease-protected single-strand DNA oligonucleotides and silencing of the mismatch repair protein, $\mathrm{MSH} 2$, enhances the replication of corrected cells following gene editing. J Gene Med. 2009;11(3):267-274.

42. Olsen PA, Randøl M, Luna L, Brown T, Krauss S. Genomic sequence correction by single-stranded DNA oligonucleotides: role of DNA synthesis and chemical modifications of the oligonucleotide ends. J Gene Med. 2005;7(12):1534-1544.

43. Huen MS, Li XT, Lu LY, Watt RM, Liu DP, Huang JD. The involvement of replication in single stranded oligonucleotide-mediated gene repair. Nucleic Acids Res. 2006;34(21):6183-6194.

44. Aarts M, te Riele H. Parameters of oligonucleotide-mediated gene modification in mouse ES cells. J Cell Mol Med. 2009;14(6B): 1657-1667.

45. Radecke S, Radecke F, Peter I, Schwarz K. Physical incorporation of a single-stranded oligodeoxynucleotide during targeted repair of a human chromosomal locus. J Gene Med. 2006;8(2):217-228.
46. Bertoni C, Morris GE, Rando TE. Strand bias in oligonucleotide-mediated dystrophin gene editing. Hum Mol Genet. 2005;14(2):221-233.

47. Ferrara L, Kmiec EB. Targeted gene repair activates Chk1 and Chk2 and stalls replication in corrected cells. DNA Repair (Amst). 2006;5(4): 422-431.

48. Liu C, Wang Z, Huen MS, Lu LY, Liu DP, Huang JD. Cell death caused by single-stranded oligodeoxynucleotide-mediated targeted genomic sequence modification. Oligonucleotides. 2009;19(3):281-286.

49. Li WJ, Laurencin CT, Caterson EJ, Tuan RS, Ko FK. Electrospun nanofibrous structure: a novel scaffold for tissue engineering. J Biomed Mater Res. 2002;60(4):613-621.

50. Bhardwaj N, Kundu SC. Electrospinning: a fascinating fiber fabrication technique. Biotechnol Adv. 2010;28(3):325-347.

51. Reneker DH, Chun I. Nanometre diameter fibres of polymer, produced by electrospinning. Nanotechnology. 1996;7:216-223.

52. Shin YM, Hohman MM, Brenner MP, Rutledge GC. Experimental characterization of electrospinning: the electronically forced jet and instabilities. Polymer. 2001;42:9955-9967.

53. Kwon IK, Kidoaki S, Matsuda T. Electrospun nano- to microfiber fabrics made of biodegradable copolyesters: structural characteristics, mechanical properties and cell adhesion potential. Biomaterials. 2005;26(18):3929-3939.

54. Christopherson GT, Song H, Mao HQ. The influence of fiber diameter of electrospun substrates on neural stem cell differentiation and proliferation. Biomaterials. 2009;30(4):556-564.

55. Cardwell RD, Dahlgren LA, Goldstein AS. Electrospun fibre diameter, not alignment, affects mesenchymal stem cell differentiation into the tendon/ligament lineage. J Tissue Eng Regen Med. Epub October 5, 2012.

56. Xie J, Willerth SM, Li X, et al. The differentiation of embryonic stem cells seeded on electrospun nanofibers into neural lineages. Biomaterials. 2009;30(3):354-362.

57. Yang X, Chen X, Wang H. Acceleration of osteogenic differentiation of preosteoblastic cells by chitosan containing nanofibrous scaffolds. Biomacromolecules. 2009;10(10):2772-2778.
International Journal of Nanomedicine

\section{Publish your work in this journal}

The International Journal of Nanomedicine is an international, peerreviewed journal focusing on the application of nanotechnology in diagnostics, therapeutics, and drug delivery systems throughout the biomedical field. This journal is indexed on PubMed Central, MedLine, CAS, SciSearch $\AA$, Current Contents ${ }^{\circledR} /$ Clinical Medicine,

\section{Dovepress}

Journal Citation Reports/Science Edition, EMBase, Scopus and the Elsevier Bibliographic databases. The manuscript management system is completely online and includes a very quick and fair peer-review system, which is all easy to use. Visit http://www.dovepress.com/ testimonials.php to read real quotes from published authors. 